\title{
Battery Energy Storage System Information Modeling Based on IEC 61850
}

\author{
Nan Wang1, Wei Liang2, Yanan Cheng1, Yunfei $\mathbf{M u}^{3}$ \\ ${ }^{1}$ State Grid Tianjin Economic Research Institute, Tianjin, China \\ ${ }^{2}$ State Grid Tianjin Electric Power Research Institute, Tianjin, China \\ ${ }^{3}$ Key Laboratory of Smart Grid of Ministry of Education, Tianjin University, Tianjin, China \\ Email: 13920692601@163.com
}

Received November 2013

\begin{abstract}
This paper discourses the typical ways to access system of the battery energy storage system. To realize the battery energy storage system based on IEC 61850, hierarchical information architecture for battery energy storage system is presented, the general design and implementation methods for device information model are elaborated, and the communication methods of the architecture are proposed. Example of battery energy storage system information model based on IEC 61850 tests that the battery energy storage system information architecture established is feasible.
\end{abstract}

\section{Keywords}

IEC 61850; Battery Energy Storage System; Information Modeling

\section{Introduction}

Using battery energy storage system in the power system can effectively implement demand-side management, reduce the peak-valley differences between day and night, and strengthen the self-regulating nature of peakvalley load in the regional power grid; Enhance the security and stability of large power grids and power quality levels, improve transmission capacity, and increase reliability; Promote renewable energy assessing to grids large-scaled. Meanwhile, the battery energy storage system is also an important part of the smart grid. Therefore, energy storage technologies applying in power system will be the development trend of the future power grid [1-3].

Currently, most of the battery energy storage system manufacturers defined device information specifications by their own way, and there were many different types of communication interfaces, which made the battery energy storage system subject to many constraints in information integration, operation control and scheduling management [4]. IEC 61850 uses object-oriented modeling techniques and flexible and scalable communication architecture, has good device characteristics self-describing capability, can meet the requirements of openness and interoperability. IEC 61850 Ed1 aims at communication networks and systems in substations; Ed2 extends coverage to the hydropower, distributed energy and substation automation, etc. which can provide effectively technical means to plug and play device integration, flexible expansion capabilities and interoperability of the information model, and other important functions in the battery energy storage system, reduces the difficulty and 
cost of system communication and control interfaces standardization work.

\section{Typical Battery Energy Storage System Accessing System Mode}

According to different functions in power system, battery energy storage system can be classified into different groups, such as the peak and valley battery energy storage system, peak shifting and frequency regulation battery energy storage system, new energy assessing battery energy storage system, and backup power battery energy storage system, etc. [5]. Different functional battery energy storage systems have different mode assessing to power system, which assess by either substations or power plants.

\subsection{Constitutions of the Battery Energy Storage System}

The battery energy storage system consists of a step-up transformer and a number of storage branches. Each storage branch contains a low voltage breaker and a storage unit. The storage unit is the basic unit of battery energy storage system, which consists of battery pack (BP), battery management system (BMS), and power conversion system (PCS). The constitution of battery energy storage system is shown in Figure 1.

\subsection{Access to Power System by Substation}

The wiring of battery energy storage system assessing to power system by substation is shown in Figure 2. The battery energy storage system connects to $10 \mathrm{kV}$ bus in the $110 \mathrm{kV}$ or $35 \mathrm{kV}$ substation through the circuit breaker [6,7]. In the valley period, the battery energy storage system runs on storing energy state, which guarantees

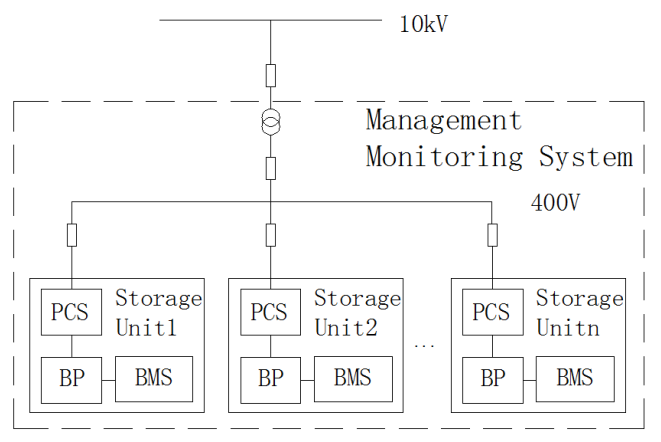

Figure 1. Constitution of the battery energy storage system.

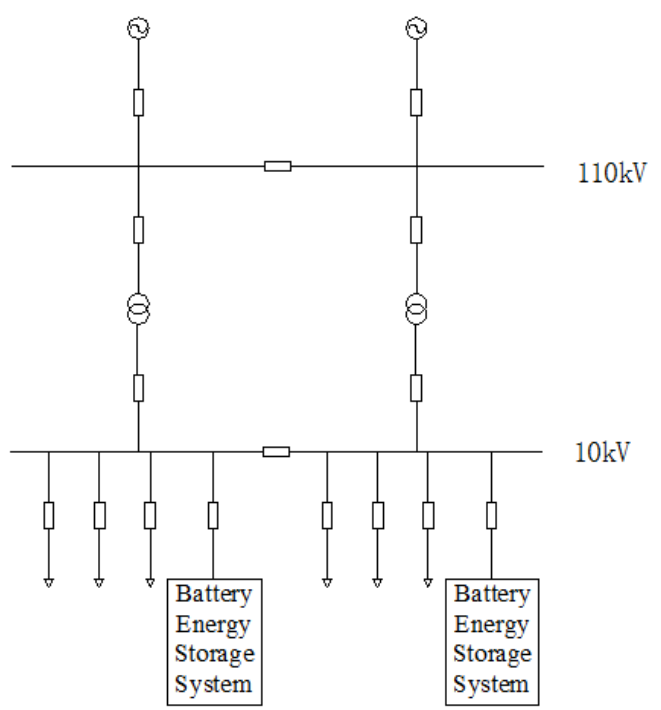

Figure 2. Access to power system by substation. 
the load consumption; in the peak period, the battery energy storage system runs on releasing energy state, which guarantees the reliable power supply. Meanwhile, the battery energy storage system can provide power to the important load as backup power when the high voltage side of the substation shutdowns, which improves reliability of power grid.

\subsection{Access to Power System by Power Plant}

Since the gradual depletion of fossil fuels and the greenhouse effect caused by the growing phenomenon of global warming, renewable energy has attracted great attention. However, intermittent renewable energy, such as wind energy and solar energy, is not continuous and stable. The battery energy storage system is used with renewable energy generation systems to improve the imbalance of the renewable energy power generation system and electric power load, the quality of power supply, and the renewable energy power system stability. The wiring of battery energy storage system assessing to power system by power plant is shown in Figure 3.

\section{Information Structure Design of the Battery Energy Storage System}

The information structure of the battery energy storage system can be classified into three layers, including the device layer, the bay layer and the management layer. The information structure of the battery energy storage system is shown in Figure 4.

\subsection{The Device Layer}

The device layer is the basic of the battery energy storage system; it is composed of physical equipment, including battery pack, DC breaker, DC current convertor, measuring and monitoring equipment, AC breaker and step-up transformer, etc. The measuring and monitoring equipment, such as current transformer, potential transformer, is in charge of battery energy storage system measuring and operating data monitoring, which provides data support for real-time analysis of battery energy storage system. The AC breaker and step-up transformer connect the battery energy storage system with the external power grid.

\subsection{The Bay Layer}

The bay layer is the bridge of information exchange between device layer and management layer. On the one

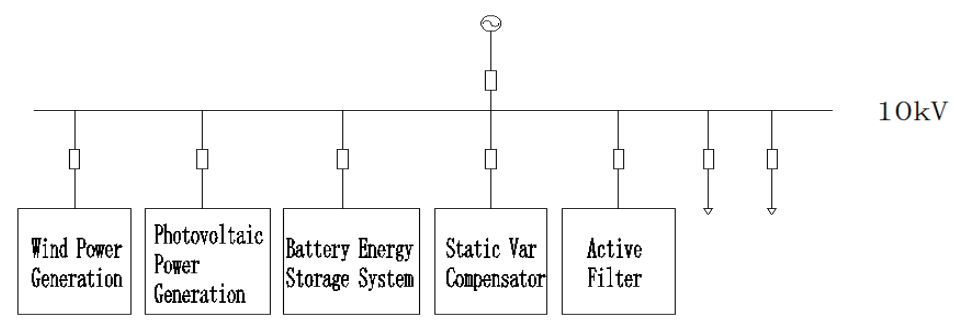

Figure 3. Access to power system by power plant.

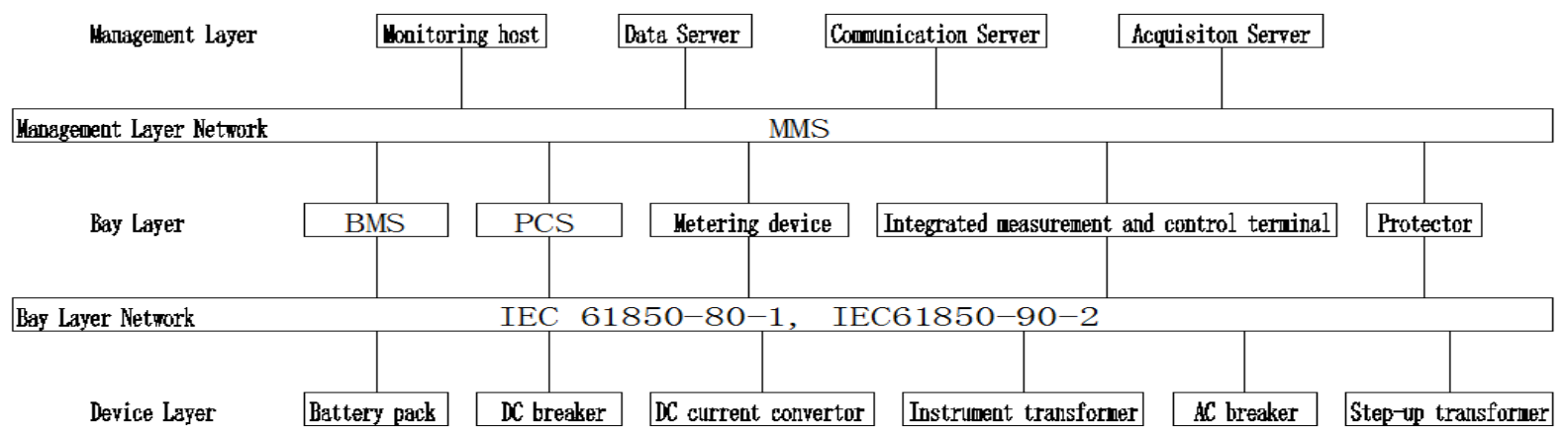

Figure 4. Information structure of the battery energy storage system. 
hand, the bay layer receives measurement data and operating status information collected by the device layer, preprocesses the data and exchanges them with the management layer; on the other hand, it receives, analyzes and delivers the control information of the management layer, realizes the management of the device. The devices in the bay layer include battery management system (BMS), power conversion system (PCS), integrated measurement and control terminal and protectors. They typically have the extended human-computer interaction interface, which are convenient for parameters configuration and operation debugging. The BMS mainly completes the function of battery monitoring, running alarm, self-diagnosis and parameter management, is able to display the battery running state, and realizes the function of storage and query of historical data. The PCS realizes battery energy storage system connecting to the grid or islanding operation according to the orders that sent by either the management layer or the bay layer. Integrated measurement and control terminal realizes the function of data collecting and control orders sending. The protectors realize the function of fault protection and status alarm of the battery energy storage system.

\subsection{The Management Layer}

The management layer is consisted of the monitoring host, operator station and telemechanism communication device, provides the man-machine interface, realizes the function of managing and controlling the device layer and the bay layer device, formats the substation monitoring\& management center, and communicates with the remote monitoring \& controlling center.

\section{The Design of Battery Energy Storage System Information Model and Communication Methods}

The battery energy storage system uses the information model based on IEC 61850 in order to realize large capacity data monitoring. Basic model structures and communication methods of the power system device follow the IEC 61850 standard Part 5, various IED device configuration descriptions follow the IEC61850 standard Part 6, the information model of automatic control, monitoring, protection and other functions follow IEC 61850 standard Part 7-4. The IEC 61850 standard (Ed.2) Part 7-420 defines the information model of distributed energy. The information model of the battery energy storage system follows the above standards. The devices that haven't meet IEC 61850 standard can be equipped with the appropriate IED to complete rapid configuration and integration of the information model [8-10].

The packets exchanged between the device layer and the bay layer including trip command, alarm signal, meter and operational data collected by sensors. The device layer communicates with the bay layer through IEDs. The BMS monitors the battery cell voltage, temperature and alarm. The PCS uses monitoring data and system control strategies to achieve closed-loop controlling of converter. Information exchange follows IEC 61850 standard Part 80-1 \& 90-2, uses fiber Ethernet to transmit data based on TCP/IP protocol in order to ensure that the communication network has high reliability between the device layer and the bay layer.

The packets exchanged between the bay layer and the management layer include automatic control data, protector value, time synchronization messages and control command packets, which have high requirements for information transmission security and real-time communication, can communicate by fiber optic network.

\section{Examples of Battery Energy Storage System Information Modeling Based on IEC 61850}

The information model of AC breaker and step-up transformer can use the model designed in ordinary substation. The information model of the battery energy storage system based on IEC 61850 is shown in Figure 5 . Main logical nodes are listed in Table $\mathbf{1 .}$

\section{Summary}

The openness and interoperability of IEC 61850 makes communication and control interface standardization of battery energy storage system feasible. This paper discourses the typical accessing ways of the battery energy storage system. To realize the battery energy storage system based on IEC 61850, hierarchical information architecture for battery energy storage system is presented, the general design and implementation methods for device information model are elaborated, and the communication methods of the architecture are proposed. 


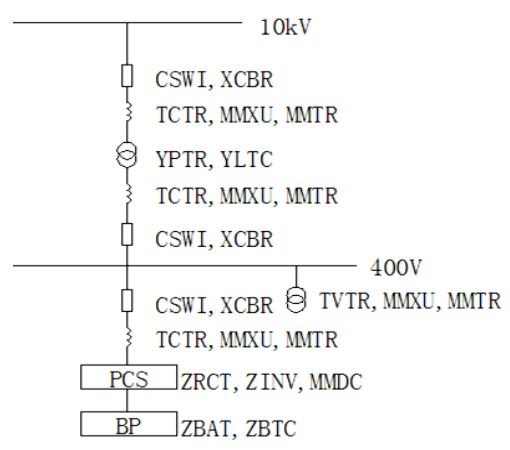

Figure 5. Information model of the battery energy storage system based on IEC 61850.

Table 1. Logical nodes and corresponding function.

\begin{tabular}{lcc}
\hline Logical node & corresponding function & Explanation \\
\hline ZBAT & Battery systems & Remote monitoring and control battery systems \\
ZBTC & Battery charger & Remote monitoring and control battery chargers \\
ZRCT & Rectifier & Define the characteristics of rectifier \\
ZINV & Inverter & Define the characteristics of inverter \\
MMDC & AC measurement & DC voltage, current, power, and resistance \\
MMXU & AC breaker controller & AC voltage, current, active and reactive power \\
CSWI & AC breaker & Describe the controller for operation of AC breaker \\
XCBR & Meter & Define the characteristics of AC breaker \\
MMTR & Transformer & Meter information \\
YPTR & Tap changer & Define the characteristics of transformer \\
YLTC & Voltage transformer & Define the characteristics of tap changer \\
TVTR & Current transformer & Define the characteristics of voltage transformer \\
TCTR & Define the characteristics of current transformer
\end{tabular}

Example of battery energy storage system information modeling based on IEC 61850 tests that the battery energy storage system information architecture established is feasible.

\section{References}

[1] Zhang, W.L., Qiu, M. and Lai, X.K. (2008) Application of Energy Storage Technologies in Power Grids. Power System Technology, 32, 1-9.

[2] (2011) Electrical Energy Storage. IEC, Geneva.

[3] Rastler, D. (2010) Electricity Energy Storage Technology Options: A White Paper Primer on Applications, Costs, and Benefits. Electric Power Research Institute (EPRI), Palo Alto.

[4] Deng, W., Pei, W. and Qi, Z.P. (2013) Microgrid Information Exchange Based on IEC 61850. Automation of Electric Power Systems, 37, 6-11.

[5] Zhou, L., Huang, Y. and Guo, K. (2011) A Survey of Energy Storage Technology for Micro Grid. Power System Protection and Control, 39,147-152.

[6] Lu, Z.G., Wang, K. and Liu, Y. (2013) Research and Application of Megawatt Scale Lithium ion Battery Energy Storage Station and Key Technology. Automation of Electric Power Systems, 37, 65-69, 127.

[7] Jin, Y.D., Song, Q., Chen, J.H., et al. (2010) Power Conversion System of Large Scaled Battery Energy Storage. Elec- 
tric Power, 43, 16-20.

[8] Cao, J.W., Wan, Y.X. and Tu, G.Y. (2013) Information System Architecture for Smart Grids. Chinese Journal of Computers, 36, 143-167.

[9] Huang, X.M. (2012) Information Modeling and Distributed Execution Mechanism for Flywheel Energy Storage System in Micro Grid. Power System and Clean Energy, 28, 54-63.

[10] Wang, D.W., Di, J. and Zhang, C.M. (2012) Information Modeling and Implementation for Status Monitoring IED in Substation Based on IEC 61850. Automation of Electric Power Systems, 36, 81-86. 